\title{
A Note on An Almost Contanct Metric Manifold with A Type of Semi-symmetric Non-metric Connection
}

\author{
Hülya Bağdatlı Yılmaz
}

(Communicated by Kazım İLARSLAN)

\begin{abstract}
In this paper, we focus on an almost contact metric manifold admitting a type of semi-symmetric nonmetric connection. We find the expression for the curvature tensor of such a manifold. Furhermore, we study the properties of the curvature tensor and the projective curvature tensor.
\end{abstract}

Keywords: Almost contact metric manifold; Semi-symmetric non-metric connection; Curvature tensor.

AMS Subject Classification (2010): 53B15.

\section{Introduction}

An odd dimensional differentiable manifold $M$ of class $C^{\infty}$ is said to have an almost contact structure by Gray [9] if the structural group of its tangent bundle reduces to $U(n) \times 1$. In 1960, Sasaki [15] showed that the notions of an almost contact structure and a $(\phi, \xi, \eta)$ - structure satisfying certain conditions are equivalent. There are many different types of almost contact structures defined in the literature such as cosymplectic, Sasakian, almost cosymplectic, quasi Sasakian, normal, $\alpha$-Kenmotsu, $\alpha$-Sasakian, trans-Sasakian, etc... [2, 3, 11, 13].

Let $\left(M^{n}, g\right)$ be an $n(=2 m+1)$-dimensional Riemannian manifold of class $C^{\infty}$. Let there exist in $M^{n}$ a 1-form $\eta$, a vector field $\xi$ and a vector valued linear function $\phi$ such that

$$
\phi^{2}=-X+\eta(X) \xi
$$

for any vector field $X$. Then $M^{n}$ is called an almost contact manifold and the system $(\phi, \xi, \eta)$ is called an almost contact structure to $M^{n}$.

From (1.1), it follows [3]

$$
\phi \xi=0, \quad \eta(\phi X)=0, \quad \eta(\xi)=1 .
$$

If the Riemannian metric $g$ in $M^{n}$ satisfies

$$
g(\phi X, \phi Y)=g(X, Y)-\eta(X) \eta(Y),
$$

for any vector fields $X$ and $Y$ in $M^{n}$, then $\left(M^{n}, g\right)$ is called an almost contact metric manifold and $g$ is called a compatible metric [15]. In view of (1.2) and (1.3), we get

$$
g(\xi, Y)=\eta(Y)
$$

and

Received : 19-11-2017, Accepted : 01-04-2018

${ }^{0}$ This article is the written version of author's plenary talk delivered on August 15-18, 2017 at 6th International Eurasian Conference on Mathematical Sciences and Applications (IECMSA 2017) in Budapest, Hungary. 


$$
g(X, \phi Y)=g(\phi X, Y) .
$$

The notion of semi-symmetric linear connection on a differentiable manifold was introduced by Friedmann and Schouten [8]. Let $\bar{\nabla}$ be a linear connection in an $n$-dimensional Riemannian manifold with Riemannian metric $g$. A linear connection $\bar{\nabla}$ on a Riemannian manifold $M^{n}$ is called a semi-symmetric connection if the torsion tensor $T$ of the connection $\bar{\nabla}$

$$
T(X, Y)=\bar{\nabla}_{X} Y-\bar{\nabla}_{Y} X-[X, Y]
$$

satisfies

$$
T(X, Y)=\pi(Y) X-\pi(X) Y,
$$

where $\pi$ is a 1 -form. The connection $\bar{\nabla}$ is a metric connection if there is a Riemannian metric $g$ in $M^{n}$ such that $\bar{\nabla} g=0$, otherwise it is non-metric. In 1932, Hayden [10] defined a semi- symmetric metric connection on a Riemannaian manifold and Yano [17] developed it. Several authors such as Pravonovich [14], Agashe and Chafle [1], Liang [12] and Sengupta, De and Binh [16] introduced a semi-symmetric non-metric connection in different ways and this connection is studied by many authors [7, 16, 18], etc.... Recently, Chaubey and Ojha [4,5] defined a new type of semi-symmetric non-metric connection in an almost contact metric manifold. In 2011, These authors [6] studied some properties of a semi-symmetric non-metric connection in a Kenmotsu manifold.

In the present paper we consider a semi-symmetric non-metric connection in the sense of Agashe and Chafle [1] and define a semi-symmetric non-metric connection in an almost contact metric manifold by identifying the 1 -form $\pi$ of (1.7) with a 1 - form $\eta$, i.e., by setting

$$
T(X, Y)=\eta(Y) X-\eta(X) Y
$$

and deal with almost contact manifolds admitting a type of semi symmetric non-metric connection $\bar{\nabla}$ satisfying the condition (1.8) and

$$
\left(\bar{\nabla}_{X} T\right)((Y, Z)=\delta(X) T(Y, Z)+w(X) g(Y, Z) \rho,
$$

where $\delta$ and $w$ are non-zero 1 -forms defined by

$$
\delta(X)=g(X, P), w(X)=g(X, \rho)
$$

and the 1-form $w$ satisfies

$$
w(R(X, Y) Z)=0 \text {. }
$$

In Section 3 we find the expression for curvature tensor of $\bar{\nabla}$ and deduce some properties of the curvature tensor. It is proved that if the curvature tensor of $\bar{\nabla}$ vanishes, the $1-$ form $\eta$ of the manifold is closed, the vector field $\xi$ is irrotational and the integral curves of $\xi$ are geodesics. In Section 4 we deal with the projective curvature tensor of $\bar{\nabla}$ and we obtain that if the Ricci tensor of $\bar{\nabla}$ is symmetric, the projective curvature tensors of the manifold with respect to the Levi-Civita connection and the semi symmetric connection are equal. Next we prove that if the Ricci tensor of $\bar{\nabla}$ vanishes, then the projective curvature tensor of the manifold is equal to the curvature tensor of the manifold with respect to the semi symmetric non-metric connection. Finally, if the curvature tensor of $\bar{\nabla}$ vanishes, we show that it is projectively flat, its scalar curvature tensor $r$ is not zero and the vector field $\xi$ is a Ricci principal direction with corresponding eigen value $r$ and we also obtain the expressions of the curvature tensor $R$ and the Ricci tensor $S$ of this manifold.

\section{Preliminaries}

The relation between the semi-symmetric non-metric connection $\bar{\nabla}$ and the Levi-Civita connection $\nabla$ of $\left(M^{n}, g\right)$ is defined in the following form by Agashe and Chafle [1]

$$
\bar{\nabla}_{X} Y=\nabla_{X} Y+\pi(Y) X
$$

where $\pi$ is a 1 -form.

Further, a relation between the curvature tensors $R$ and $\bar{R}$ of type $(1,3)$ of the connections $\nabla$ and $\bar{\nabla}$ respectively are given by [1] 


$$
\bar{R}(X, Y) Z=R(X, Y) Z+\alpha(X, Z) Y-\alpha(Y, Z) X
$$

where $\alpha$ is a tensor field of type $(0,2)$ defined by

$$
\alpha(X, Y)=\left(\nabla_{X} \pi\right)(Y)-\pi(X) \pi(Y)=\left(\bar{\nabla}_{X} \pi\right)(Y) .
$$

Contracting (2.2) we have

$$
\bar{S}(Y, Z)=S(Y, Z)+(1-n) \alpha(Y, Z)
$$

where $\bar{S}$ and $S$ denote the Ricci tensors of the semi-symmetric non-metric connection and Levi-Civita connection, respectively.

\section{Almost contact metric manifolds admitting a special type of semi-symmetric non-metric connection}

In this section we consider an almost contact metric manifold admitting semi-symmetric non-metric connection whose torsion tensor $T$ is given by (1.8) and it satisfies (1.9). Then, from (1.8), contracting over $X$, we get

$$
\left(C_{1}^{1} T\right)(Y)=(n-1) \eta(Y) .
$$

From (3.1), it follows that

$$
\left(\bar{\nabla}_{X} C_{1}^{1} T\right)(Y)=(n-1)\left(\bar{\nabla}_{X} \eta\right)(Y) .
$$

Contracting (1.9) and using (3.1), we get

$$
\left(\bar{\nabla}_{X} C_{1}^{1} T\right)(Y)=(n-1) \delta(X) \eta(Y)+w(X) w(Y) .
$$

Using (3.2), from (3.3), we obtain

$$
\left(\bar{\nabla}_{X} \eta\right)(Y)=\delta(X) \eta(Y)+\frac{1}{n-1} w(X) w(Y) .
$$

Since $\bar{\nabla}_{X} Y=\nabla_{X} Y+\eta(Y) X$, from $\left(\bar{\nabla}_{X} \eta\right)(Y)=\bar{\nabla}_{X} \eta(Y)-\eta\left(\bar{\nabla}_{X} Y\right)$, it follows that

$$
\left(\bar{\nabla}_{X} \eta\right)(Y)=\left(\nabla_{X} \eta\right)(Y)-\eta(X) \eta(Y) .
$$

Combining (3.4) and (3.5), we have

$$
\left(\nabla_{X} \eta\right)(Y)=\eta(X) \eta(Y)+\delta(X) \eta(Y)+\frac{1}{n-1} w(X) w(Y),
$$

in virtue of (2.3) and (3.5), we get

$$
\alpha(X, Y)=\delta(X) \eta(Y)+\frac{1}{n-1} w(X) w(Y) .
$$

Now, using (2.2) and (3.7), the expression of the curvature tensor $\bar{R}$ with respect to the connection $\bar{\nabla}$ can be written as

$$
\begin{aligned}
\bar{R}(X, Y) Z & =R(X, Y) Z+\left\{\delta(X) \eta(Z)+\frac{1}{n-1} w(X) w(Z)\right\} Y \\
& -\left\{\delta(Y) \eta(Z)+\frac{1}{n-1} w(Y) w(Z)\right\} X
\end{aligned}
$$

Thus we can state the following thorem:

Theorem 3.1. The curvature tensor with respect to $\bar{\nabla}$ of an almost contact metric manifold admitting the semi-symmetric non-metric connection whose torsion tensor $T$ is given by (1.8) satisfies (1.9) is of the form (3.8). 
Replacing $X$ with $Y$ in (3.8), from (3.8), it is clear that

$$
\bar{R}(X, Y) Z=-\bar{R}(Y, X) Z .
$$

From (3.8), we get

$$
\begin{aligned}
\bar{R}(X, Y) Z+\bar{R}(Y, Z) X+\bar{R}(Z, X) Y & =\{\delta(Z) \eta(Y)-\delta(Y) \eta(Z)\} X \\
& +\{\delta(X) \eta(Z)-\delta(Z) \eta(X)\} Y \\
& +\{\delta(Y) \eta(X)-\delta(X) \eta(Y)\} Z
\end{aligned}
$$

This is the first Bianchi identity with respect to $\bar{\nabla}$.

Again from (3.8), we get by contracting $X$

$$
\bar{S}(Y, Z)=S(Y, Z)-(n-1) \delta(Y) \eta(Z)-w(Y) w(Z) .
$$

In (3.11) we put $Y=Z=e_{i}, 1 \leq i \leq n$, where $\left\{e_{i}\right\}$ is an orthonormal basis of the tangent space at each point of the manifold. Then summing over $i$ we get

$$
\bar{r}=r-(n-1) \delta(\xi)-w(\rho)
$$

where $\bar{r}$ and $r$ denote the scalar curvatures of this manifold with respect to $\bar{\nabla}$ and $\nabla$, respectively.

From (3.11), it follows that $\bar{S}$ is symmetric if and only if

$$
\delta(Y) \eta(Z)=\delta(Z) \eta(Y) .
$$

Putting $Z=\xi$ in (3.13) and using (1.2), we get

$$
\delta(Y)=a \eta(Y)
$$

where $a=\delta(\xi)$.

Now let us assume that $\bar{S}$ is symmetric. Then (3.10) and (3.14) we get

$$
\bar{R}(X, Y) Z+\bar{R}(Y, Z) X+\bar{R}(Z, X) Y=0 .
$$

Conversely, we assume that (3.15) holds, then in virtue of (3.10) we have

$$
\{\delta(Z) \eta(Y)-\delta(Y) \eta(Z)\} X+\{\delta(X) \eta(Z)-\delta(Z) \eta(X)\} Y+\{\delta(Y) \eta(X)-\delta(X) \eta(Y)\} Z=0 .
$$

Contracting $X$, from (3.16), it follows that

$$
\delta(Y) \eta(Z)-\delta(Z) \eta(Y)=0
$$

Hence by (3.13), $\bar{S}$ is symmetric.

Thus, we can state the following theorem:

Theorem 3.2. A necessary and sufficient condition for the Ricci tensor of an almost contact metric manifold with respect to the semi-symmetric non-metric connection whose torsion tensor is given by (1.8) satisfies (1.9) to be symmetric is

$$
\bar{R}(X, Y) Z+\bar{R}(Y, Z) X+\bar{R}(Z, X) Y=0 .
$$

From (3.8) and (3.14), it follows that

$$
\begin{aligned}
\bar{R}(X, Y) Z & =R(X, Y) Z+\left\{a \eta(X) \eta(Z)+\frac{1}{n-1} w(X) w(Z)\right\} Y \\
& -\left\{a \eta(Y) \eta(Z)+\frac{1}{n-1} w(Y) w(Z)\right\} X .
\end{aligned}
$$

We now define a covariant curvature tensor $\bar{R}$ of type $(0,4)$ by 


$$
\bar{R}(X, Y, Z, V)=g(\bar{R}(X, Y) Z, V) .
$$

In virtue of (3.19), from (3.18), we have

$$
\begin{aligned}
\bar{R}(X, Y, Z, V) & =R(X, Y, Z, V)+\left\{a \eta(X) \eta(Z)+\frac{1}{n-1} w(X) w(Z)\right\} g(Y, V) \\
& -\left\{a \eta(Y) \eta(Z)+\frac{1}{n-1} w(Y) w(Z)\right\} g(X, V) .
\end{aligned}
$$

If $\bar{R}=0$, then we get

$$
\begin{aligned}
R(X, Y, Z, V) & =\left\{a \eta(Y) \eta(Z)+\frac{1}{n-1} w(Y) w(Z)\right\} g(X, V) \\
& -\left\{a \eta(X) \eta(Z)+\frac{1}{n-1} w(X) w(Z)\right\} g(Y, V)
\end{aligned}
$$

and

$$
S(Y, Z)=(n-1) a \eta(Z) \eta(Y)+w(Z) w(Y) .
$$

Thus, from (3.21) and (3.22), it follows that

$$
R(X, Y, Z, V)=\frac{1}{(n-1)}\{S(Y, Z) g(X, V)-S(X, Z) g(Y, V)\} .
$$

Putting $V=\rho$ in (3.23), we obtain

$$
w(R(X, Y) Z)=\frac{1}{(n-1)}\{S(Y, Z) w(X)-S(X, Z) w(Y)\} .
$$

Using (1.11) and (3.22), we get

$$
a\{\eta(Y) w(X)-\eta(X) w(Y)\}=0 .
$$

Since $\delta$ is a non-zero 1-form, from the above equation, it follows that

$$
\eta(Y) w(X)=\eta(X) w(Y) .
$$

Hence by (1.2) and (3.26), we have

$$
w(Y)=b \eta(Y)
$$

where $b=w(\xi)$.

From (3.6), (3.14) and (3.27), it follows that

$$
\left(\nabla_{X} \eta\right)(Y)=\left[1+a+\frac{b^{2}}{n-1}\right] \eta(X) \eta(Y) .
$$

Therefore, we get

$$
\left(\nabla_{X} \eta\right)(Y)=\left(\nabla_{Y} \eta\right)(X)
$$

Hence by (3.29), we find that the 1-form $\eta$ is closed. From (3.14) and (3.27), it follows that the 1 -forms $\delta$ and $w$ are also closed.

Furthermore, using (3.29), we have

$$
g\left(Y, \nabla_{X} \xi\right)=g\left(X, \nabla_{Y} \xi\right)
$$


which implies that the vector field $\xi$ is irrotational. Since $g(\xi, \xi)=1$, then

$$
g\left(X, \nabla_{\xi} \xi\right)=0
$$

that is, $\nabla_{\xi} \xi=0$ which implies that the integral curves of $\xi$ are geodesics.

Hence we can state:

Theorem 3.3. If an almost contact metric manifold admits a semi-symmetric non-metric connection whose torsion tensor $T$ is given by (1.8) satisfies (1.9) and whose curvature tensor vanishes, then the 1 - form $\eta$ of the manifold is closed, the vector field $\xi$ is irrotational and the integral curves of $\xi$ are geodesics.

\section{Projective curvature tensor}

The projective curvature tensor of type $(1,3)$ of an almost contact metric manifold with respect to the semisymmetric non-metric connection is defined by

$$
\bar{P}(X, Y) Z=\bar{R}(X, Y) Z-\frac{1}{n-1}\{\bar{S}(Y, Z) X-\bar{S}(X, Z) Y\} .
$$
(4.1)

If, in particular, $\bar{S}$ is symmetric, then we already have $\delta(Y)=\delta(\xi) \eta(Y)$. Thus, using (3.8) and (3.11), we get from

$$
\bar{P}(X, Y) Z=P(X, Y) Z,
$$

where $P(X, Y) Z$ is the projective curvature tensor of the manifold with respect to the Levi-Civita connection defined as

$$
P(X, Y) Z=R(X, Y) Z-\frac{1}{n-1}\{S(Y, Z) X-S(X, Z) Y\}
$$

So, we have

Theorem 4.1. If the Ricci tensor of an almost contact metric manifold with respect to the semi-symmetric non-metric connection whose torsion tensor $T$ is given by (1.8) satisfies (1.9) is symmetric, then the projective curvature tensors of the manifold with respect to the Levi-Civita connection and the semi symmetric non-metric connection are equal.

In virtue of (4.2) and (4.3) we can state the following:

Corollary 4.1. If the Ricci tensor of an almost contact metric manifold with respect to the semi-symmetric non-metric connection whose torsion tensor $T$ is given by (1.8) satisfies (1.9) is symmetric, then it is satisfied the conditions:

i $\bar{P}(X, Y) Z+\bar{P}(Y, Z) X=0$,

ii $\bar{P}(X, Y) Z+\bar{P}(Y, Z) X+\bar{P}(Z, X) Y=0$.

Next, if in particular $\bar{S}=0$, then from (4.1) and (4.2) we have

$$
P(X, Y) Z=\bar{R}(X, Y) Z \text {. }
$$

So we can state:

Theorem 4.2. If the Ricci tensor of an almost contact metric manifold with respect to the semi-symmetric non-metric connection whose torsion tensor $T$ is given by (1.8) satisfies (1.9) vanishes, then the projective curvature tensor of the manifold is equal to the curvature tensor of the manifold with respect to the semi symmetric non-metric connection.

If $\bar{R}=0$, then from (3.18) and (3.27), it follows that

$$
R(X, Y) Z=\left[a-\frac{b^{2}}{n-1}\right] \eta(Z)\{\eta(X) Y-\eta(Y) X\} .
$$

Contracting (4.5) we get

$$
S(Y, Z)=\left[b^{2}-a(n-1)\right] \eta(Y) \eta(Z) .
$$


Further contraction yields

$$
r=b^{2}-a(n-1) .
$$

Let $L$ be the symmetric linear operator such that

$$
S(Y, Z)=g(L Y, Z) .
$$

Thus from (4.6) and (4.8), we get

$$
g(L Y, Z)=r \eta(Y) \eta(Z) .
$$

Putting $Y=\xi$ in (4.9) and using (1.2), we obtain

$$
L \xi=r \xi .
$$

Hence, using (4.3) we have the following theorem:

Theorem 4.3. If an almost contact metric manifold admits a semi-symmetric non-metric connection $\bar{\nabla}$ with the vector field $\xi$ whose curvature tensor $\bar{R}$ vanishes and whose torsion tensor $T$ is given by (1.8) satisfies (1.9), then

i The curvature tensor $R$ and the Ricci tensor $S$ of this manifold are respectively given by (4.5) and (4.6). Its scalar curvature is also not zero.

ii It is projectively flat.

iii The vector field $\xi$ is a Ricci principal direction with corresponding eigen value $r$.

Moreover, from (1.2), (1.4), (4.5) and (4.6) we can state the following theorem:

Theorem 4.4. If an almost contact metric manifold admits a semi-symmetric non-metric connection whose torsion tensor $T$ is given by (1.8) satisfies (1.9) and whose curvature tensor vanishes, then we have

i $\eta(R(X, Y) Z)=0$,

ii $R(X, Y) \xi=\left[a-\frac{b^{2}}{n-1}\right]\{\eta(X) Y-\eta(Y) X\}$,

iii $S(Y, \xi)=r \eta(Y)$.

\section{Acknowledgement}

This research was supported by BAPKO at Marmara University, Grant No: FEN-D-090517-0299.

\section{References}

[1] Agashe, N. S. and Chafle, M. R., A semi-symmetric non-metric connection on a Riemannian manifold, Indian J. Pure Appl. Math 23 (1992), 399-409.

[2] Blair, D. E., The theory of quasi-Sasakian structures J. Diff. Geom. 1 (1967), 331-345.

[3] Blair, D. E., Contact manifolds in Riemannian geometry, Lecture Notes in Math., 0509, Springer-Verlag, Berlin 1976.

[4] Chaubey, S. K., On semi symmetric non-metric connection, Prog. of Math., 41-42(2007), 11-20.

[5] Chaubey, S. K. and Ojha, R. H., On semi symmetric non-metric connection and quarter symmetric connections, Tensor N. S., 70 (2008),No:2, 202-213.

[6] Chaubey, S. K. and Ojha, R. H., On semi symmetric non-metric connection, Filomat, 25:4(2011) , 19-27.

[7] De, U. C. and Biswas, S. C., On a type of semi symmetric non-metric connection on a Riemannian manifold, Pub. De L'institut Math. Nouvelle serie tome 61, 1997, 90-96. 
[8] Friedmann, A. and Schouten, J. A., Über die Geometric der holbsymmetrischen Übertragurgen, Math. Z., 21(1924), 211-233.

[9] Gray, J., Some global properties of contact structures, Ann. of Math., 69(1959), 421-450.

[10] Hayden, H. A., Subspaces of space with torsion, Proc. London Math. Soc., 34(1932), 27-50.

[11] Janssens, D. and Vanhecke, L., Almost contact structures and curvature tensors, Kodai Math. J., 4 (1981), 1-27.

[12] Liang,Y., On semi-symmetric recurrent metric connection, Tensor N. S. 55 (1994), 107-102.

[13] Oubina, J., New classes of almost contact metric structures, Publicationes Mathematicae, 32 (1985), 187-193.

[14] Pravonovic, M., On pseudo symmetric semi-symmetric connection, Pub. De L'Institu Math., Nouvelle Serie, 18(32) (1975), 157-164.

[15] Sasaki, S., On diff. manifolds with certain structures which are closely related to almost contact structure I, Tohoku Math. J., 12(1960), 459-476.

[16] Sengupta, J., De, U. C. and Binh, T. Q., On a type of semi symmetric non-metric connection on a Riemannian manifold, Indian J. Pure Appl. Math.,31(12) (2000),1659-1670.

[17] Yano, K., On semi-symmetric metric connections, Revue Roumania De Math. Pures Appl. 15 (1970), 1579-1586.

[18] Yilmaz, H. B., On weakly symmetric manifolds with a type of semi-symmetric non-metric connection, Ann. Polonici Math., 102.3 (2011),301-308.

\section{Affiliation}

HÜLYA BAĞDATLI YILMAZ

AdDRESS: Marmara University, Dept. of Sciences and Letters, 34722, Istanbul-Turkey.

E-MAIL: hbagdatli@marmara.edu.tr

ORCID ID: $0000-0003-2568-7577$ 